\title{
Sobre la innovación y el impacto de la investigación
}

\author{
Norberto Malpica-González
}

Associate Professor - Electronics Medical Image Analysis and Biometry Lab Universidad Rey Juan Carlos

La investigación es uno de los pilares básicos del trabajo en la Universidad. Pero desde hace algunos años, el término investigación nunca aparece solo. Primero surgió el término I+D (investigación y desarrollo), indicando que a la investigación básica se añadía la creación y el desarrollo de productos y procesos, incluyendo de ese modo el trabajo tecnológico e ingenieril en el concepto de investigación universitaria. Recientemente, se amplió el acrónimo a l+D+i, añadiendo la palabra innovación al concepto, indicando que la innovación no es mero desarrollo. El término innovación lo encontramos hoy en día por todas partes. En la prensa, en los programas de las Universidades... Y este año, la COVID-19 ha hecho que hablemos aún más de innovación. Hay que pensar nuevas formas de hacer las cosas, nuevos productos, nuevos procesos.

Si buscamos en el diccionario, la primera acepción de innovación es "acción y efecto de innovar", siendo innovar "mudar o alterar algo, introduciendo novedades". Esta es la idea general de innovación que usamos comúnmente. Cualquier procedimiento que introduzca algo nuevo en un producto o un proceso, sería innovación. Pero hay una segunda acepción de la palabra, que es más interesante desde el punto de vista académico: "Creación o modificación de un producto, y su introducción en un mercado." La idea del mercado es crucial, ya que permite crear nuevas empresas y generar empleo, que era en definitiva el objetivo de introducir esa tercera letra en I+D+i. La innovación englobaría, pues, el proceso completo de transferencia de tecnología.

Un primer paso para fomentar la transferencia tecnológica ha sido promover la obtención de patentes. Hace ya tiempo que las Universidad y los organismos de evaluación de la actividad investigadora han añadido las patentes en sus baremos, para fomentar que los académicos solicitemos patentes, además de redactar artículos científicos.

La última palabra que ha aparecido en los últimos años unida a la innovación es 'emprendimiento'. Esperamos de los egresados universitarios no solo que busquen trabajo en una compañía existente, sino que creen su propia compañía. Por ello, las Universidades están fomentando la creación de empresas de base tecnológica a partir de los resultados de la investigación. Estas empresas, denominadas spin-off, pueden ser creadas por egresados, o ser aventuras conjuntas en que los propios docentes e investigadores se involucren a tiempo parcial. En muchos países, el número de empresas de base tecnológica creadas se está convirtiendo en una de las métricas que miden la calidad de una Universidad.

Un primer enfoque para la creación de empresas es el análisis de las patentes registradas por la Universidad o centro de investigación, con la intención de dar salida a alguna de ellas mediante el modelo de negocio adecuado. Pero este modelo no suele funcionar, ya que estas patentes no siempre surgen de una necesidad externa. Muchas surgen de una mejora en el estado del arte de una tecnología, pero no provienen de un análisis de necesidades del usuario ni de la detección de un mercado potencial. Y es aquí donde se hace necesario un cambio de paradigma. Tanto la innovación como la investigación académica no siempre están basadas en necesidades reales. El modelo de investigación de la Universidades consiste en incorporar estudiantes de doctorado o investigadores postdoctorales a líneas de investigación existentes, y el sistema de financiación de la investigación premia a los grupos que ya son expertos en una línea determinada. Siendo, por tanto, muy difícil conseguir financiación para ideas nuevas. En muchos casos, el investigador o profesor que se incorpora a una línea no vuelve a hacerse preguntas de fondo, que podrían reorientar su investigación.

Existen técnicas de innovación que están muy extendidas en entornos de diseño y emprendimiento, como el Design Thinking (Brown, 2008), que describe un proceso completo de innovación desde el análisis de la necesidad hasta el modelo de negocio, pasando por el diseño de soluciones y el prototipado. En el caso de la innovación biomédica, esta metodología ha sido adaptada con el nombre de Biodesign, con resultados muy interesantes (Schwartz, 2016). 
Este tipo de técnicas permiten pasar del modelo de "tecnología en busca de una aplicación" a una innovación basada en el análisis de necesidades, y pueden aplicarse también a la investigación universitaria. Un investigador que se incorpore a un nuevo grupo o línea de investigación debería preguntarse: ¿Cuál es la necesidad de esta línea de investigación?, ¿Cuál es el impacto social, científico o clínico de la misma?, ¿Qué necesidades importantes podría solucionar con mi investigación? También es importante la multidisciplinariedad de la investigación en todo el ciclo de la misma. No basta con incorporar a los potenciales usuarios de una tecnología una vez desarrollada, sino que sería interesante contar con una visión amplia de distintos expertos a lo largo de todo el proyecto de investigación, para analizar el desarrollo completo desde diversos puntos de vista. Esta es una práctica habitual actualmente en innovación, en la línea de los métodos ágiles de desarrollo software (Williams, 2003).

En definitiva, la incorporación de técnicas de innovación y agilidad en la definición y desarrollo de la investigación puede dar lugar a nuevas líneas de trabajo con un mayor impacto científico, económico y social.

\section{Referencias}

Brown T. Design thinking. Harvard Business Review, 2008;86(6):84-92.

Williams L, Cockburn A. Agile software development: it's about feedback and change. IEEE computer, 2003;36(6):39-43.

Schwartz JG, Kumar UN, Azagury DE, Brinton TJ, Yock PG. Needs-based innovation in cardiovascular medicine: The Stanford Biodesign Process. JACC: Basic to Translational Science, 2016;1(6):541-547. 
https://doi.org/10.22579/20112629.624

\title{
On innovation and the impact of research
}

\author{
Norberto Malpica-González
}

Associate Professor - Electronics Medical Image Analysis and Biometry Lab Universidad Rey Juan Carlos

Research is one of the basic pillars of work at university level; however, the term research has never appeared alone for some years now. Firstly, the term research and development $(\mathrm{R}+\mathrm{D})$ appeared, indicating that the creation and development of products and processes had been added to basic research, thereby including technological and engineering work within the concept of university research. The acronym has recently been extended to read RD\&l, adding the word innovation to the concept, indicating that innovation is not merely development. The term innovation can be found everywhere today, in the press, in university programmes and now, throughout this year, COVID-19 has made us talk even more about innovation. One has to think about new ways of doing things, new products, new processes.

Looking in the dictionary, the first meaning for innovation would probably be, "the action and effect of innovating," to innovate being, "changing or altering something, introducing novelties." This would be the commonly used overall idea of innovation; any procedure introducing something new into a product or process would be innovation. However, the word has a second meaning which is more interesting from an academic point of view: "creating or modifying a product and introducing it to a (target) market." The market concept is crucial since it enables new companies to be created and jobs to be generated, ultimately the object of introducing such third letter into RD\&l; innovation thus encompasses the whole technology transfer $(T T)$ process.

A first step in promoting $\Pi \mathrm{T}$ has been to encourage researchers to obtain patents; universities and research evaluation organisms have added patents to their scales for some time now to encourage academics to apply for patents, as well as writing scientific articles.

'Entrepreneurship' has been the latest word to be linked with innovation in recent years. We expect university graduates to seek work in an existing company; nowadays we also expect them to create their own. Universities are thus encouraging the creation of technology-based companies based on research results. Such companies, called spin-offs, can be created by graduates or be joint ventures in which teachers and researchers themselves become involved as part-time participants. The amount of technology-based companies created in many countries is becoming one of the metrics used for measuring university quality.

Analysing the patents registered by a university or research centre represents a first approach to creating a company, aimed at enabling some of them to enter a targeted market via a suitable business model. However, such model usually does not work since these patents do not always arise from external need; many arise from an improvement regarding a particular technology's state of the art rather than analysing user needs or detecting a potential market. This is where a paradigm shift is necessary; innovation, just like academic research, is not always based on real need. Universities' research models usually consist of incorporating PhD students or postdoc researchers into existing lines of research and the system for financing research rewards groups which are already considered experts regarding a determined line, thereby making it very difficult to obtain funding for new ideas. A researcher or teacher joining an established line of research often desists from posing in-depth questions as this could lead to reorientating such research.

Some innovation techniques have become widespread regarding design and entrepreneurship settings, such as design thinking (Brown, 2008) which describes a complete innovation process from analysing a particular need to the business model, through solution design and prototyping. Biomedical innovation has been adapted under the name of 'biodesign', leading to very interesting results (Schwartz, 2016). 
Such techniques enable moving on from a "technology seeking an application" model to a "need assessment-based innovation" model which can also be used for university research. A researcher joining a new group or line of research should ask her/himself the following questions: "What is the need for/purpose of this line of research?" "What is its social, scientific or clinical impact?" "Which important needs could be resolved through my research?" Research's multidisciplinarity throughout its whole cycle is also important; even though potential users of a particular technology can become incorporated once it has been developed, it would be interesting to have different experts' broader vision throughout an entire research project for analysing its complete development from various points of view. This is common practice which is currently used regarding innovation, in line with agile software development methods (Williams, 2003).

In short, incorporating innovation and agility techniques into the definition and development of research can lead to new lines of work/research having greater scientific, economic and social impact.

\section{References}

Brown T. Design thinking. Harvard Business Review, 2008;86(6):84-92.

Williams L, Cockburn A. Agile software development: it's about feedback and change. IEEE computer, 2003;36(6):39-43.

Schwartz JG, Kumar UN, Azagury DE, Brinton TJ, Yock PG. Needs-based innovation in cardiovascular medicine: The Stanford Biodesign Process. JACC: Basic to Translational Science, 2016;1(6):541-547. 
https://doi.org/10.22579/20112629.624

\title{
Sobre a inovação e o impacto da pesquisa
}

\author{
Norberto Malpica-González
}

Professor Associado - Laboratório de Biometria e Análise de Imagens Médicas Eletrônicas Universidad Rey Juan Carlos

\begin{abstract}
A pesquisa é um dos pilares básicos do trabalho da Universidade. Mas, há alguns anos, o termo pesquisa nunca aparece só. Primeiramente, surgiu o termo P + D (pesquisa e desenvolvimento), indicando que a pesquisa básica foi agregada à criação e ao desenvolvimento de produtos e processos, incluindo o trabalho tecnológico e de engenharia no conceito de pesquisa universitária. Recentemente, a sigla para P + D + i foi ampliada, acrescentando a palavra inovação ao conceito, indicando que inovação não é mero desenvolvimento. O termo inovação é encontrado em todos os lugares hoje. Na imprensa, nos programas universitários ... E este ano, o COVID-19 fez-nos falar ainda mais em inovação. Você tem que pensar em novas maneiras de fazer as coisas, novos produtos, novos processos.
\end{abstract}

Se olharmos no dicionário, o primeiro significado de inovação é "ação e efeito de inovar", sendo inovar "mudando ou alterando algo, introduzindo novidades". Esta é a ideia geral de inovação que comumente usamos. Qualquer procedimento que introduza algo novo em um produto ou processo seria inovação. Mas existe um segundo significado da palavra, que é mais interessante do ponto de vista acadêmico: "Criação ou modificação de um produto e sua introdução no mercado." A ideia de mercado é crucial, uma vez que permite a criação de novas empresas e a geração de empregos, que foi em última análise o objetivo de introduzir aquela terceira letra na I + D + i. A inovação abrangeria, assim, todo o processo de transferência de tecnologia.

Um primeiro passo para promover a transferência de tecnologia foi promover a obtenção de patentes. Há algum tempo, universidades e órgãos de avaliação de pesquisas vêm adicionando patentes às suas escalas, para estimular os acadêmicos a solicitarem patentes, além de escreverem artigos científicos.

A última palavra que apareceu nos últimos anos ligada à inovação é 'empreendedorismo'. Esperamos que os graduados não apenas busquem trabalho em uma empresa já existente, mas criem sua própria empresa. Por isso, as Universidades estão promovendo a criação de empresas de base tecnológica a partir dos resultados das pesquisas. Essas empresas, chamadas spin-offs, podem ser criadas por graduados ou ser joint ventures nas quais os próprios professores e pesquisadores se envolvam em meio período. Em muitos países, o número de empresas de base tecnológica criadas está se tornando uma das métricas que medem a qualidade de uma Universidade.

Uma primeira abordagem para a criação de empresas é a análise das patentes registradas pela Universidade ou centro de pesquisa, com a intenção de liberar algumas delas através do modelo de negócio adequado. Mas esse modelo geralmente não funciona, uma vez que essas patentes nem sempre surgem de uma necessidade externa. Muitos surgem de uma melhoria no estado da arte de uma tecnologia, mas não vêm de uma análise das necessidades do usuário ou da detecção de um mercado potencial. E é aqui que uma mudança de paradigma é necessária. Tanto a inovação quanto a pesquisa acadêmica nem sempre se baseiam em necessidades reais. O modelo de pesquisa das Universidades consiste em incorporar doutorandos ou pós-doutorandos às linhas de pesquisa existentes, e o sistema de financiamento à pesquisa recompensa grupos que já são especialistas em determinada linha. Sendo, portanto, muito difícil conseguir financiamento para novas ideias. Em muitos casos, o pesquisador ou professor que ingressa em uma linha não volta a fazer perguntas aprofundadas, o que poderia reorientar sua pesquisa.

Existem técnicas de inovação muito difundidas em ambientes de design e empreendedorismo, como o Design Thinking [Brown, 2008], que descreve um processo de inovação completo desde a análise da necessidade ao modelo de negócio, 
passando pelo design de soluções e a prototipado. No caso da inovação biomédica, essa metodologia foi adaptada sob o nome de Biodesign, com resultados muito interessantes [Schwartz, 2016].

Esses tipos de técnicas nos permitem passar do modelo "tecnologia em busca de uma aplicação" para uma inovação baseada na análise de necessidades, podendo também ser aplicadas à pesquisa universitária. Um pesquisador que ingressa em um novo grupo ou linha de pesquisa deve se perguntar: Qual a necessidade dessa linha de pesquisa? Qual é o impacto social, científico ou clínico dela? Que necessidades importantes ele poderia resolver com minha investigação? A multidisciplinaridade da pesquisa também é importante ao longo de seu ciclo. Não basta incorporar potenciais usuários de uma tecnologia uma vez desenvolvida, mas seria interessante ter uma visão ampla de diferentes especialistas ao longo de todo

o projeto de pesquisa, para analisar o desenvolvimento completo sob diferentes pontos de vista. Atualmente, essa é uma prática comum em inovação, alinhada aos métodos ágeis de desenvolvimento de software [Williams, 2003].

Em suma, a incorporação de técnicas de inovação e agilidade na definição e no desenvolvimento da pesquisa pode levar a novas linhas de trabalho com maior impacto científico, econômico e social.

\section{Referências}

Brown T. Design thinking. Harvard Business Review, 2008;86(6):84-92.

Williams L, Cockburn A. Agile software development: it's about feedback and change. IEEE computer, 2003;36(6):39-43.

Schwartz JG, Kumar UN, Azagury DE, Brinton TJ, Yock PG. Needs-based innovation in cardiovascular medicine: The Stanford Biodesign Process. JACC: Basic to Translational Science, 2016;1(6):541-547. 